Echocardiography measurement for left ventricular function revealed mean for ejection fraction was $48 \%$ and mean for fractional shortening was $26 \%$. Pericardial effusion was the most common seen in DCM patients.

Conclusion: The most common underlying disease for DCM in Saiful Anwar General Hospital due to renal disease, the age were more than five years old and almost all the children had moderate malnutrition.

doi:10.1016/j.ijcard.2017.09.101

\section{Implications of High-Sensitivity Cardiac Troponin I in Cardiology Clinical Practice}

C.S. Khaw ${ }^{\mathrm{a}}$, L.L.C. Loretta ${ }^{\mathrm{b}}$, H. Amirul Fadhli ${ }^{\mathrm{b}}$, M. Azzlee ${ }^{\mathrm{b}}$, Y. John ${ }^{\mathrm{a}}$, H.S. Lim ${ }^{\mathrm{a}}$, N. Madzlan ${ }^{\mathrm{a}}$, M.Z. Noor Najihah ${ }^{\mathrm{b}}$, Y. Nasyitah ${ }^{\mathrm{a}}$, R. Shahirab ${ }^{\mathrm{b}}$, Z.Y. Siow ${ }^{\mathrm{a}}$, A.S. Mohd Zailani ${ }^{\mathrm{b}}$, M.A. Nor Hanim ${ }^{\mathrm{a}}$, N.Z. Khiewa ${ }^{\mathrm{a}}$, Y.L. Cham ${ }^{\mathrm{a}}$, S. Asri, ${ }^{\mathrm{a}, \mathrm{d}}$, C.Y. Voon $^{\mathrm{a}}$, Y.Y. Oon ${ }^{\mathrm{a}}$, K.T. Koh ${ }^{\mathrm{a}}$, K.H. Ho ${ }^{\mathrm{a}}$, C.T. Tan ${ }^{\mathrm{a}}$, A.Y.Y. Fong ${ }^{\mathrm{a}, \mathrm{c}}$, T.K. Ong ${ }^{\mathrm{a}}$

${ }^{a}$ Department of Cardiology, Sarawak Heart Centre, Kota Samarahan, Malaysia ${ }^{b}$ Department of Emergency, Sarawak Heart Centre, Kota Samarahan, Malaysia ${ }^{c}$ Clinical Research Centre, Sarawak General Hospital, Kuching, Malaysia

${ }^{d}$ Faculty of Medicine and Health Sciences, University Malaysia Sarawak, Kota Samarahan, Malaysia

Background: High sensitivity troponin (hsTn) has better sensitivity for myocardial tissue injury detection compared to standard troponin assays, despite lower diagnostic specificity and lack of hsTn assay standardization.

Objectives: To examine implications of introducing hsTnI in clinical practice.

Methods: We retrospectively collected information of patients presented to a single tertiary cardiac referral centre with suspected acute coronary syndrome (ACS), who had $\geq 1$ hsTnI sample (Abbott ARCHITECT STAT), from 1st June 2016-17th August 2016. Upper range limit (URL), i.e. $99^{\text {th }}$ percentile, was defined as $34.2 \mathrm{ng} / \mathrm{L}, 15.6 \mathrm{ng} / \mathrm{L}$ and $26.2 \mathrm{ng} / \mathrm{L}$ for male, female and both gender ("overall") respectively. Patients were divided into 4 groups - Group 1: hsTnURL but $<3$ times URL, Group 3: hsTn between 3-5 times URL and Group 4: hsTn $>5$ times URL.

Results: Data from 366 patients was analysed: 227(62.0\%), $34(9.3 \%), 13(3.6 \%), 92(25.1 \%)$ in Group 1, 2, 3 and 4 respectively. In Group 1 to 4 , the proportion of ACS was $8.8 \%, 38.0 \%, 53.8 \%, 82.6 \%$ and proportion of MI were $0.8 \%, 29.4 \%, 53.8 \%$. $82.6 \%$. By using $>5$ times URL as cut-off, hsTnI has higher specificity and PPV, but lower sensitivity and NPV in diagnosis of ACS \{(sensitivity:0.66, specificity:0.0.94, PPV0.83, NPV0.85), with ROC curve AUC:0.896, $\mathrm{p}<0.001$, 95\% CI:0.861-0.931\}. Our analysis showed serial paired hsTn samples increase the PPV of hsTn to detect ACS. There was no significant difference between using hsTn URL "overall" or "gender-specific" for ACS diagnosis. Kaplan-Meier analysis showed 30-day all-cause mortality in the group with maximal hsTn value $>$ URL is significant higher $(p<0.001)$. Multiple-logistic regression showed that URL of hsTn was an independent variable for 30-day all-cause mortality $(\mathrm{p}<0.001)$.

Conclusions: Introducing hsTnI has led to the recognition of a large proportion of patients with minor cardiac troponin increases (above URL of $99^{\text {th }}$ percentile but $<5$ times URL), the majority of whom do not have ACS or MI. There is no significant difference in using "overall" and "gender-specific" URL in diagnosing ACS. Using 5 times above URL and serial hsTn will increase PPV to detect ACS. Maximal HsTn value $>99$ th percentile is independently associated with 30-day all-cause mortality.

doi:10.1016/j.ijcard.2017.09.102

\section{Orthostatic Hypotension in the Malaysian Elders Longitudinal} Research (MELOR)

N.I. Saedon ${ }^{\mathrm{a}}$, J. Frith ${ }^{\mathrm{b}}$, C.H. Goh ${ }^{\mathrm{a}}$, S.B. Shahrul ${ }^{\mathrm{a}}$, A.V. Chin ${ }^{\mathrm{a}}$, H.M. Khor ${ }^{\mathrm{a}}$, M.P. Tan ${ }^{\mathrm{a}}$

${ }^{a}$ Department of Medicine, Faculty of Medicine, University of Malaya, Kuala Lumpur ${ }^{b}$ Institute for Ageing and Health, Campus for Ageing and Vitality, Newcastle University, UK

Background: Orthostatic hypotension $(\mathrm{OH})$ is defined as a systolic (SBP) drop $>20 \mathrm{mmHg}$ or diastolic $\mathrm{BP}(\mathrm{DBP})>10 \mathrm{mmHg}$ within 3 minutes of standing. Little is known about the characteristics of individuals with $\mathrm{OH}$ or its relevance in the older population.

Objective: To evaluate the haemodynamic characteristics of individuals with $\mathrm{OH}$ using data from a cohort study involving community dwelling older adults.

Methods: The Malaysian Elders Longitudinal Research (MELoR) study is longitudinal study of ageing involving adults aged 55 years and above selected from three parliamentary constituencies within the Klang Valley, Kuala Lumpur. Demographic information and baseline characteristics were collected during a home-based computer assisted interview. Subsequently patients were then invited to the local teaching hospital for health assessments. Postural blood pressure change was assessed during five minutes' supine rest followed by three minutes of standing using non-invasive continuous blood pressure measurements (Task Force, CNSystems, Austria).

Results: Haemodynamic data was available for 1245 participants. 936 (75\%) fulfilled consensus criteria for $\mathrm{OH}$. Participants with $\mathrm{OH}$ were slightly older (68.8(7.2) VS 67.9 (7.2) years; $\mathrm{p}=0.105)$. Women were significantly more likely to have $\mathrm{OH}$ than men ( $53 \%$ vs $47 \%, \mathrm{p}=0.001$ ). Individuals with $\mathrm{OH}$ had significantly higher baseline supine SBP (114.4(22.4) vs 109.9 (21.0), $\mathrm{p}=0.002$ ) but there was no significant difference in DBP (69.4(16.4) vs 67.5(14.2), $\mathrm{p}=0.079$ ). Individual with $\mathrm{OH}$ were also more likely to report a history of hypertension (471(72.2) vs $181(27.8), \mathrm{p}=0.012$ ) and atrial fibrillation (43(87.8)vs 6(12.2), $\mathrm{p}=$ 0.025).

Conclusions: Three out of four individuals aged 55 years and over fulfilled the criteria of orthostatic hypotension when their blood pressure was measured using continuous non-invasive monitoring. The clinical significance of $\mathrm{OH}$ in this population remains unclear. Future research will now focus on determining factors which determine clinically significant $\mathrm{OH}$.

doi:10.1016/j.ijcard.2017.09.103

Body Mass Index and Waist Circumference as Predictive Factors in the Development of Acute Coronary Syndrome in Young Adults

R.E.F. Raja Shariff, N. Chua, R.N. Khir, K.S. Ibrahim, M.K. Mohd Arshad, C.W. Lim, J. Rizwal, E. Abdul Rahman, H. Aiza, Z.O. Ibrahim, S. Kasim

Faculty of Medicine, UiTM Sg Buloh, Malaysia

Background: Cardiovascular disease (CVD) is the primary cause of death globally. In Malaysia, the mean age of acute coronary syndrome (ACS) is 56 years. However, younger patients lacking common risk factors are more frequently being diagnosed with ACS. As these risk factors are often present at later years, there may be an underestimation of CVD risk and subsequent lack of attempts in employing primary prevention measures in this group of patients. Fortunately, there is greater appreciation for alternative variables in predicting CVD risk. Body mass index (BMI) is a predictor variable in the British-developed 'Q-RISK2' model, which helps guide the prescription of statin therapy in at-risk groups. Likewise, waist 